PROCEEDINGS OF THE

AMERICAN MATHEMATICAL SOCIETY

Volume 140, Number 10, October 2012, Pages 3377-3384

S 0002-9939(2012)11191-8

Article electronically published on February 22, 2012

\title{
SYMMETRIC TENSOR RANK WITH A TANGENT VECTOR: A GENERIC UNIQUENESS THEOREM
}

\author{
EDOARDO BALLICO AND ALESSANDRA BERNARDI
}

(Communicated by Irena Peeva)

\begin{abstract}
Let $X_{m, d} \subset \mathbb{P}^{N}, N:=\left(\begin{array}{c}m+d \\ m\end{array}\right)-1$, be the order $d$ Veronese embedding of $\mathbb{P}^{m}$. Let $\tau\left(X_{m, d}\right) \subset \mathbb{P}^{N}$ be the tangent developable of $X_{m, d}$. For each integer $t \geq 2$ let $\tau\left(X_{m, d}, t\right) \subseteq \mathbb{P}^{N}$ be the join of $\tau\left(X_{m, d}\right)$ and $t-2$ copies of $X_{m, d}$. Here we prove that if $m \geq 2, d \geq 7$ and $t \leq 1+\left\lfloor\left(\begin{array}{c}m+d-2 \\ m\end{array}\right) /(m+1)\right\rfloor$, then for a general $P \in \tau\left(X_{m, d}, t\right)$ there are uniquely determined $P_{1}, \ldots, P_{t-2} \in$ $X_{m, d}$ and a unique tangent vector $\nu$ of $X_{m, d}$ such that $P$ is in the linear span of $\nu \cup\left\{P_{1}, \ldots, P_{t-2}\right\}$; i.e. a degree $d$ linear form $f$ (a symmetric tensor $T$ of order $d$ ) associated to $P$ may be written as
\end{abstract}

$$
f=L_{t-1}^{d-1} L_{t}+\sum_{i=1}^{t-2} L_{i}^{d}, \quad\left(T=v_{t-1}^{\otimes(d-1)} v_{t}+\sum_{i=1}^{t-2} v_{i}^{\otimes d}\right)
$$

with $L_{i}$ linear forms on $\mathbb{P}^{m}$ ( $v_{i}$ vectors over a vector field of dimension $m+1$ respectively), $1 \leq i \leq t$, that are uniquely determined (up to a constant).

\section{INTRODUCTION}

In this paper we want to address the question of the uniqueness of a particular decomposition for certain given homogeneous polynomials. An analogous question can be rephrased in terms of uniqueness of a particular tensor decomposition of certain given symmetric tensors. In fact, given a homogeneous polynomial $f$ of degree $d$ in $m+1$ variables defined over an algebraically closed field $\mathbb{K}$, there is an obvious way to associate a symmetric tensor $T \in S^{d}\left(V_{\mathbb{K}}\right)$, with $\operatorname{dim}\left(V_{\mathbb{K}}\right)=m+1$, to the form $f$. We will always work over an algebraically closed field $\mathbb{K}$ such that $\operatorname{char}(\mathbb{K})=0$. Fix integers $m \geq 2$ and $d \geq 3$. Let $j_{m, d}: \mathbb{P}^{m} \hookrightarrow \mathbb{P}^{N}, N:=\left(\begin{array}{c}m+d \\ m\end{array}\right)-1$, be the order $d$ Veronese embedding of $\mathbb{P}^{m}$ and set $X_{m, d}:=j_{m, d}\left(\mathbb{P}^{m}\right)$ (we often write $X$ instead of $\left.X_{m, d}\right)$. Let $\mathbb{K}\left[x_{0}, \ldots, x_{m}\right]_{d}$ be the polynomial ring of homogeneous degree $d$ polynomials in $m+1$ variables over $\mathbb{K}$ and let $V_{\mathbb{K}}^{*}$ be the dual space of $V_{\mathbb{K}}$. Since obviously $\mathbb{P}^{m} \simeq \mathbb{P}\left(\mathbb{K}\left[x_{0}, \ldots, x_{m}\right]_{1}\right) \simeq \mathbb{P}\left(V_{\mathbb{K}}^{*}\right)$, an element of the Veronese variety $X_{m, d}$ can be interpreted either as the projective class of a $d$-th power of a linear form $L \in \mathbb{K}\left[x_{0}, \ldots, x_{m}\right]_{1}$ or as the projective class of a symmetric tensor $T \in S^{d}\left(V_{\mathbb{K}}^{*}\right) \subset\left(V_{\mathbb{K}}^{*}\right)^{\otimes d}$ for which there exists $v \in V_{\mathbb{K}}^{*}$ s.t. $T=v^{\otimes d}$.

For each integer $t$ such that $1 \leq t \leq N$ let $\sigma_{t}(X)$ denote the closure in $\mathbb{P}^{N}$ of the union of all $(t-1)$-dimensional linear subspaces spanned by $t$ points of

Received by the editors January 26, 2011 and, in revised form, April 11, 2011.

2010 Mathematics Subject Classification. Primary 14N05, 14M17.

Key words and phrases. Veronese variety, tangential variety, join, weak defectivity.

The authors were partially supported by CIRM of FBK Trento (Italy), Project Galaad of INRIA Sophia Antipolis Méditerranée (France), Institut Mittag-Leffler (Sweden), Marie Curie: Promoting Science (FP7-PEOPLE-2009-IEF), MIUR and GNSAGA of INdAM (Italy).

(C)2012 American Mathematical Society 3377

Reverts to public domain 28 years from publication 
$X$ (the $t$-secant variety of $X$ ). From this definition one can understand that the generic element of $\sigma_{t}\left(X_{m, d}\right)$ can be interpreted either as $[f]=\left[L_{1}^{d}+\cdots+L_{t}^{d}\right] \in$ $\mathbb{P}\left(\mathbb{K}\left[x_{0}, \ldots, x_{m}\right]_{d}\right)$ with $L_{1}, \ldots, L_{t} \in \mathbb{K}\left[x_{0}, \ldots, x_{m}\right]_{1}$ or as $[T]=\left[v_{1}^{\otimes d}+\cdots+v_{t}^{\otimes d}\right] \subset$ $\mathbb{P}\left(S^{d}\left(V_{\mathbb{K}}^{*}\right)\right)$ with $v_{1}, \ldots, v_{t} \in V_{\mathbb{K}}^{*}$. For a given form $f$ (or a symmetric tensor $T$ ), the minimum integer $t$ for which there exists such a decomposition is called the symmetric rank of $f$ (or of $T$ ). Finding those $v_{i}, i=1, \ldots, t$, such that $T=v_{1}^{\otimes d}+$ $\cdots+v_{t}^{\otimes d}$, with $t$ the symmetric rank of $T$, is known as the tensor decomposition problem and it is a generalization of the singular value decomposition problem for symmetric matrices (i.e. if $T \in S^{2}\left(V_{\mathbb{K}}^{*}\right)$ ). The existence and the possible uniqueness of the decompositions of a form $f$ as $L_{1}^{d}+\cdots+L_{t}^{d}$ with $t$ minimal is studied in certain cases in [6, 8], 10, 11.

Let $\tau(X) \subseteq \mathbb{P}^{N}$ be the tangent developable of $X$, i.e. the closure in $\mathbb{P}^{N}$ of the union of all embedded tangent spaces $T_{P} X, P \in X$. Obviously $\tau(X) \subseteq \sigma_{2}(X)$ and $\tau(X)$ is integral. Since $d \geq 3$, the variety $\tau(X)$ is a divisor of $\sigma_{2}(X)$ ([5. Proposition 3.2]). An element in $\tau\left(X_{m, d}\right)$ can be described both as $[f] \in \mathbb{P}\left(\mathbb{K}\left[x_{0}, \ldots, x_{m}\right]_{d}\right)$ for which there exist two linear forms $L_{1}, L_{2} \in \mathbb{K}\left[x_{0}, \ldots, x_{m}\right]_{1}$ such that $f=L_{1}^{d-1} L_{2}$ and as $[T] \in \mathbb{P}\left(S^{d}\left(V_{\mathbb{K}}^{*}\right)\right)$ for which there exist two vectors $v_{1}, v_{2} \in V_{\mathbb{K}}^{*}$ such that $T=v_{1}^{\otimes d-1} v_{2}([5,[4])$.

Fix integral positive-dimensional subvarieties $A_{1}, \ldots, A_{s} \subset \mathbb{P}^{N}, s \geq 2$. The join $\left[A_{1}, A_{2}\right]$ is the closure in $\mathbb{P}^{N}$ of the union of all lines spanned by a point of $A_{1}$ and a different point of $A_{2}$. If $s \geq 3$, define inductively the join $\left[A_{1}, \ldots, A_{s}\right]$ by the formula $\left[A_{1}, \ldots, A_{s}\right]:=\left[\left[A_{1}, \ldots, A_{s-1}\right], A_{s}\right]$. The join $\left[A_{1}, \ldots, A_{s}\right]$ is an integral variety and $\operatorname{dim}\left(\left[A_{1}, \ldots, A_{s}\right]\right) \leq \min \left\{N, s-1+\sum_{i=1}^{s} \operatorname{dim}\left(A_{i}\right)\right\}$. The integer $\min \{N, s-1+$ $\left.\sum_{i=1}^{s} \operatorname{dim}\left(A_{i}\right)\right\}$ is called the expected dimension of the join $\left[A_{1}, \ldots, A_{s}\right]$. Obviously $\left[A_{1}, \ldots, A_{s}\right]=\left[A_{\sigma(1)}, \ldots, A_{\sigma(s)}\right]$ for any permutation $\sigma:\{1, \ldots, s\} \rightarrow\{1, \ldots, s\}$. The secant variety $\sigma_{t}(X), t \geq 2$, is the join of $t$ copies of $X$. For each integer $t \geq 3$ let $\tau(X, t) \subseteq \mathbb{P}^{N}$ be the join of $\tau(X)$ and $t-2$ copies of $X$. We recall that $\min \{N, t(m+1)-2\}$ is the expected dimension of $\tau(X, t)$, while $\min \{N, t(m+1)-1\}$ is the expected dimension of $\sigma_{t}(X)$. In the range of triples $(m, d, t)$ we will meet in this paper, both $\tau(X, t)$ and $\sigma_{t}(X)$ have the expected dimensions and hence $\tau(X, t)$ is a divisor of $\sigma_{t}(X)$. An element in $\tau\left(X_{m, d}, t\right)$ can be described both as $[f] \in$ $\mathbb{P}\left(\mathbb{K}\left[x_{0}, \ldots, x_{m}\right]_{d}\right)$ for which there exist linear forms $L_{1}, \ldots, L_{t} \in \mathbb{K}\left[x_{0}, \ldots, x_{m}\right]_{1}$ such that $f=L_{t-1}^{d-1} L_{t}+\sum_{i=1}^{t-2} L_{i}^{d}$ and as $[T] \in \mathbb{P}\left(S^{d}\left(V_{\mathbb{K}}^{*}\right)\right)$ for which there exist $v_{1}, \ldots, v_{t} \in V_{K}^{*}$ such that $T=v_{t-1}^{\otimes(d-1)} v_{t}+\sum_{i=1}^{t-2} v_{i}^{\otimes d}$.

After [3, it is natural to ask the following question.

Question 1. Assume $d \geq 3$ and $\tau(X, t) \neq \mathbb{P}^{N}$. Is a general point of $\tau(X, t)$ in the linear span of a unique set $\left\{P_{0}, P_{1}, \ldots, P_{t-2}\right\}$ with $\left(P_{0}, P_{1}, \ldots, P_{t-2}\right) \in \tau(X) \times$ $X^{t-2}$ ?

For non-weakly $(t-1)$-degenerate subvarieties of $\mathbb{P}^{N}$ the corresponding question is true by [8, Proposition 1.5]. Here we answer it for a large set of triples of integers $(m, d, t)$ and prove the following result.

Theorem 1. Fix integers $m \geq 2$ and $d \geq 6$. If $m \leq 4$, then assume $d \geq 7$. Set $\beta:=\left\lfloor\left(\begin{array}{c}m+d-2 \\ m\end{array}\right) /(m+1)\right\rfloor$. Assume $3 \leq t \leq \beta+1$. Let $P$ be a general point 
of $\tau(X, t)$. Then there are uniquely determined points $P_{1}, \ldots, P_{t-2} \in X$ and $Q \in$ $\tau(X)$ such that $P \in\left\langle\left\{P_{1}, \ldots, P_{t-2}, Q\right\}\right\rangle$; i.e. (since $d>2$ ) there are uniquely determined points $P_{1}, \ldots, P_{t-2} \in X$ and a unique tangent vector $\nu$ of $X$ such that $P \in\left\langle\left\{P_{1}, \ldots, P_{t-2}\right\} \cup \nu\right\rangle$.

In terms of homogeneous polynomials Theorem 1 may be rephrased in the following way.

Theorem 2. Fix integers $m \geq 2$ and $d \geq 6$. If $m \leq 4$, then assume $d \geq 7$. Set $\beta:=\left\lfloor\left(\begin{array}{c}m+d-2 \\ m\end{array}\right) /(m+1)\right\rfloor$. Assume $3 \leq t \leq \beta+1$. Let $P$ be a general point of $\tau(X, t)$ and let $f$ be a homogeneous degree $d$ form in $\mathbb{K}\left[x_{0}, \ldots, x_{m}\right]$ associated to $P$. Then $f$ may be written in a unique way:

$$
f=L_{t-1}^{d-1} L_{t}+\sum_{i=1}^{t-2} L_{i}^{d}
$$

with $L_{i} \in \mathbb{K}\left[x_{0}, \ldots, x_{m}\right]_{1}, 1 \leq i \leq t$.

In the statement of Theorem 2 the form $f$ is uniquely determined only up to a non-zero scalar, and (as usual in this topic) "uniqueness" may allow not only a permutation of the forms $L_{1}, \ldots, L_{t-2}$, but also a scalar multiplication of each $L_{i}$.

In terms of symmetric tensors Theorem 1 may be rephrased in the following way.

Theorem 3. Fix integers $m \geq 2$ and $d \geq 6$. If $m \leq 4$, then assume $d \geq 7$. Set $\beta:=\left\lfloor\left(\begin{array}{c}m+d-2 \\ m\end{array}\right) /(m+1)\right\rfloor$. Assume $3 \leq t \leq \beta+1$. Let $P$ be a general point of $\tau(X, t)$ and let $T \in S^{d}\left(V_{\mathbb{K}}^{*}\right)$ be a symmetric tensor associated to $P$. Then $T$ may be written in a unique way:

$$
T=v_{t-1}^{\otimes(d-1)} v_{t}+\sum_{i=1}^{t-2} v_{i}^{\otimes d}
$$

with $v_{i} \in V_{\mathbb{K}}^{*}, 1 \leq i \leq t$.

As above, in the statement of Theorem 3 the tensor $T$ and the vectors $v_{i}$ are uniquely determined only up to non-zero scalars.

To prove Theorem 11, and hence Theorems 2] and 3, we adapt the notion and the results on weakly defective varieties described in [6]. It is easy to adapt [6] to joins of different varieties instead of secant varieties of a fixed variety if a general tangent hyperplane is tangent only at one point $(7])$. However, a general tangent space of $\tau(X)$ is tangent to $\tau(X)$ along a line, not just at the point of tangency. Hence a general hyperplane tangent to $\tau(X, t), t \geq 3$, is tangent to $\tau(X, t)$ at least along a line. We prove the following result.

Theorem 4. Fix integers $m \geq 2$ and $d \geq 6$. If $m \leq 4$, then assume $d \geq 7$. Set $\beta:=\left\lfloor\left(\begin{array}{c}m+d-2 \\ m\end{array}\right) /(m+1)\right\rfloor$. Assume $t \leq \beta+1$. Let $P$ be a general point of $\tau(X, t)$. Let $P_{1}, \ldots, P_{t-2} \in X$ and $Q \in \tau(X)$ be the points such that $P \in\left\langle\left\{P_{1}, \ldots, P_{t-2}, Q\right\}\right\rangle$. Let $\nu$ be the tangent vector of $X$ such that $Q$ is a point of $\langle\nu\rangle \backslash \nu_{\text {red }}$. Let $H \subset \mathbb{P}^{N}$ be a general hyperplane containing the tangent space $T_{P} \tau(X, t)$ of $\tau(X, t)$. Then $H$ is tangent to $X$ only at the points $P_{1}, \ldots, P_{t-2}, \nu_{r e d}$, the scheme $H \cap X$ has an ordinary node at each $P_{i}$, and $H$ is tangent to $\tau(X) \backslash X$ only along the line $\langle\nu\rangle$. 


\section{Preliminaries}

Notation 1. Let $Y$ be an integral quasi-projective variety and let $Q \in Y_{\text {reg }}$. Let $\{k Q, Y\}$ denote the $(k-1)$-st infinitesimal neighborhood of $Q$ in $Y$, i.e. the closed subscheme of $Y$ with $\left(\mathcal{I}_{Q}\right)^{k}$ as its ideal sheaf. If $Y=\mathbb{P}^{m}$, then we write $k Q$ instead of $\left\{k Q, \mathbb{P}^{m}\right\}$. The scheme $\{k Q, Y\}$ will be called a $k$-point of $Y$. We also say that a 2-point is a double point, that a 3 -point is a triple point and that a 4 -point is a quadruple point.

We give here the definition of a $(2,3)$-point as it is in [5, p. 977].

Definition 1. Let $\mathfrak{q} \subset \mathbb{K}\left[x_{0}, \ldots, x_{m}\right]$ be the reduced ideal of a simple point $Q \in \mathbb{P}^{m}$, and let $l \subset \mathbb{K}\left[x_{0}, \ldots, x_{m}\right]$ be the ideal of a reduced line $L \subset \mathbb{P}^{m}$ through $Q$. We say that $Z(Q, L)$ is a $(2,3)$-point if it is the zero-dimensional scheme whose representative ideal is $\left(\mathfrak{q}^{3}+l^{2}\right)$.

Remark 1. Notice that $2 Q \subset Z(Q, L) \subset 3 Q$.

We recall the notion of weak non-defectivity for an integral and non-degenerate projective variety $Y \subset \mathbb{P}^{r}$ (see [6]). For any closed subscheme $Z \subset \mathbb{P}^{r}$ set

$$
\mathcal{H}(-Z):=\left|\mathcal{I}_{Z, \mathbb{P}^{r}}(1)\right| \text {. }
$$

Notation 2. Let $Z \subset \mathbb{P}^{r}$ be a zero-dimensional scheme such that $\{2 Q, Y\} \subseteq Z$ for all $Q \in Z_{\text {red }}$. Fix $H \in \mathcal{H}(-Z)$ where $\mathcal{H}(-Z)$ is defined in (11). Let $H_{c}$ be the closure in $Y$ of the set of all $Q \in Y_{\text {reg }}$ such that $T_{Q} Y \subseteq H$.

The contact locus $H_{Z}$ of $H$ is the union of all irreducible components of $H_{c}$ containing at least one point of $Z_{\text {red }}$.

We use the notation $H_{Z}$ only in the case $Z_{\text {red }} \subset Y_{\text {reg }}$.

Fix an integer $k \geq 0$ and assume that $\sigma_{k+1}(Y)$ does not fill up the ambient space $\mathbb{P}^{r}$. Fix a general $(k+1)$-uple of points in $Y$, i.e. $\left(P_{0}, \ldots, P_{k}\right) \in Y^{k+1}$, and set

$$
Z:=\bigcup_{i=0}^{k}\left\{2 P_{i}, Y\right\} .
$$

The following definition of weakly $k$-defective variety coincides with the one given in [6].

Definition 2. A variety $Y \subset \mathbb{P}^{r}$ is said to be weakly $k$-defective if $\operatorname{dim}\left(H_{Z}\right)>0$ for $Z$ as in (2).

In 6. Theorem 1.4], it is proved that if $Y \subset \mathbb{P}^{r}$ is not weakly $k$-defective, then $H_{Z}=Z_{\text {red }}$ and that $\operatorname{Sing}(Y \cap H)=(\operatorname{Sing}(Y) \cap H) \cup Z_{\text {red }}$ for a general $Z=$ $\bigcup_{i=0}^{k}\left\{2 P_{i}, Y\right\}$ and a general $H \in \mathcal{H}(-Z)$. Notice that $Y$ is weakly 0 -defective if and only if its dual variety $Y^{*} \subset \mathbb{P}^{r *}$ is not a hypersurface.

In [7 the same authors considered also the case in which $Y$ is not irreducible and hence its joins have as irreducible components the joins of different varieties.

Lemma 1. Fix an integer $y \geq 2$, an integral projective variety $Y, L \in \operatorname{Pic}(Y)$ and $P \in Y_{\text {reg. Set } x}:=\operatorname{dim}(Y)$. Assume $h^{0}\left(Y, \mathcal{I}_{(y+1) P} \otimes L\right)=h^{0}(Y, L)-\left(\begin{array}{c}x+y \\ x\end{array}\right)$. Fix a general $F \in\left|\mathcal{I}_{y P} \otimes L\right|$. Then $P$ is an isolated singular point of $F$.

Proof. Let $u: Y^{\prime} \rightarrow Y$ denote the blowing-up of $Y$ at $P$ and $E:=u^{-1}(P)$ the exceptional divisor. Since $\operatorname{dim}(Y)=x$, we have $E \cong \mathbb{P}^{x-1}$. Set $R:=u^{*}(L)$. For each integer $t \geq 0$ we have $u_{*}(R(-t E)) \cong \mathcal{I}_{t P} \otimes L$. Thus the push-forward $u_{*}$ 
induces an isomorphism between the linear system $|R(-t E)|$ on $Y^{\prime}$ and the linear system $\left|\mathcal{I}_{t P} \otimes L\right|$ on $Y$. Set $M:=R(-y E)$. Since $\mathcal{O}_{Y^{\prime}}(E) \mid E \cong \mathcal{O}_{E}(-1)$ (up to the identification of $E$ with $\mathbb{P}^{x-1}$ ), we have $R(-t E) \mid E \cong \mathcal{O}_{E}(t)$ for all $t \in \mathbb{N}$. Consider on $Y^{\prime}$ the exact sequence

$$
0 \rightarrow M(-E) \rightarrow M \rightarrow \mathcal{O}_{E}(y) \rightarrow 0 .
$$

Our hypothesis implies that $h^{0}\left(Y, \mathcal{I}_{y P} \otimes L\right)=h^{0}(Y, L)-\left(\begin{array}{c}x+y-1 \\ x\end{array}\right)$. Thus our assumption implies $h^{0}\left(Y^{\prime}, M(-E)\right)=h^{0}\left(Y^{\prime}, R\right)-\left(\begin{array}{c}x+y \\ x\end{array}\right)=h^{0}\left(Y^{\prime}, R\right)-\left(\begin{array}{c}x+y-1 \\ x\end{array}\right)-\left(\begin{array}{c}x+y-1 \\ x-1\end{array}\right)=$ $h^{0}\left(Y^{\prime}, M\right)-h^{0}\left(E, \mathcal{O}_{E}(y)\right)$. Thus (3) gives the surjectivity of the restriction map $\rho: H^{0}\left(Y^{\prime}, M\right) \rightarrow H^{0}\left(E,\left.M\right|_{E}\right)$. Since $y \geq 0$, the line bundle $M \mid E$ is spanned. Thus the surjectivity of $\rho$ implies that $M$ is spanned at each point of $E$. Hence $M$ is spanned in a neighborhood of $E$. Bertini's theorem implies that a general $F^{\prime} \in|M|$ is smooth in a neighborhood of $E$. Since $F$ is general and $|M| \cong\left|\mathcal{I}_{y P} \otimes L\right|, P$ is an isolated singular point of $F$.

\section{3. $\tau(X, t)$ IS NOT WEAK DEFECTIVE}

In this section we fix integers $m \geq 2$ and $d \geq 3$ and set $N=\left(\begin{array}{c}m+d \\ m\end{array}\right)-1$ and $X:=X_{m, d}$. The variety $\tau(X)$ is 0 -weakly defective, because a general tangent space of $\tau(X)$ is tangent to $\tau(X)$ along a line. Terracini's lemma for joins implies that a general tangent space of $\tau(X, t)$ is tangent to $\tau(X, t)$ at least along a line (see Remark 2). Thus $\tau(X, t)$ is weakly 0 -defective. To handle this problem and prove Theorem 1 we introduce another definition, which is tailor-made to this particular case. As in [5] we want to work with zero-dimensional schemes on $X$, not on $\tau(X)$ or $\tau(X, t)$. We consider $X=j_{m, d}\left(\mathbb{P}^{m}\right)$ and the 0 -dimensional scheme $Z \subset X$ which is the image (via $j_{m, d}$ ) of the general disjoint union of $t-2$ double points and one $(2,3)$-point of $\mathbb{P}^{m}$, in the case of [5] (see Definition 1 ). We will often work by identifying $X$ with $\mathbb{P}^{m}$, so e.g. notice that $\mathcal{H}(-\emptyset)$ is just $\left|\mathcal{O}_{\mathbb{P}^{m}}(d)\right|$.

Remark 2. Fix $P \in X$ and $Q \in T_{P} X \backslash\{P\}$. Any two such pairs $(P, Q)$ are projectively equivalent for the natural action of $\operatorname{Aut}\left(\mathbb{P}^{m}\right)$. We have $Q \in \tau(X)_{\text {reg }}$ and $T_{Q} \tau(X) \supset T_{P} X$. Set $D:=\langle\{P, Q\}\rangle$. It is well known that $D \backslash\{P\}$ is the set of all $O \in \tau(X)_{\text {reg }}$ such that $T_{Q} \tau(X)=T_{O} \tau(X)$ (e.g. use the fact that the set of all $g \in \operatorname{Aut}\left(\mathbb{P}^{m}\right)$ fixing $P$ and the line containing $P$ associated to the tangent vector induced by $Q$ acts transitively on $\left.T_{P} X \backslash D\right)$.

Definition 3. Fix a general $\left(O_{1}, \ldots, O_{t-2}, O\right) \in\left(\mathbb{P}^{m}\right)^{t-1}$ and a general line $L \subset \mathbb{P}^{m}$ such that $O \in L$. Set $Z:=Z(O, L) \cup \bigcup_{i=1}^{t-2} 2 O_{i}$. We say that the variety $\tau(X, t)$ is not drip defective if $\operatorname{dim}\left(H_{Z}\right)=0$ for a general $H \in\left|\mathcal{I}_{Z}(d)\right|$.

We are now ready for the following lemma.

Lemma 2. Fix an integer $t \geq 3$ such that $(m+1) t<n$. Let $Z_{1} \subset \mathbb{P}^{m}$ be a general union of a quadruple point and $t-2$ double points. Let $Z_{2}$ be a general union of two triple points and $t-2$ double points. Fix a general disjoint union $Z=$ $Z(O, L) \cup\left(\bigcup_{i=1}^{t-2} 2 P_{i}\right)$, where $Z(O, L)$ is a $(2,3)$-point as in Definition 1 and $O, L$ and $\left\{P_{1}, \ldots, P_{t-2}\right\} \subset \mathbb{P}^{m}$ are general. Assume $h^{1}\left(\mathbb{P}^{m}, \mathcal{I}_{Z_{1}}(d)\right)=h^{1}\left(\mathbb{P}^{m}, \mathcal{I}_{Z_{2}}(d)\right)=0$. Then:

(i) $h^{1}\left(\mathbb{P}^{m}, \mathcal{I}_{Z}(d)\right)=0$;

(ii) $\tau(X, t)$ is not drip defective;

(iii) a general $H \in \mathcal{H}(-Z)$ has an ordinary quadratic singularity at each $P_{i}$. 
Proof. Set $W:=3 O \cup\left(\bigcup_{i=1}^{t-2} 2 P_{i}\right)$. The definition of a (2,3)-point gives that $Z(O, L)$ $\subset 3 O$. Thus $Z \subset W \subset Z_{2}$. Hence $h^{1}\left(\mathbb{P}^{m}, \mathcal{I}_{Z}(d)\right) \leq h^{1}\left(\mathbb{P}^{m}, \mathcal{I}_{Z_{2}}(d)\right)=0$. Hence part (i) is proven.

To prove part (ii) of the lemma we need to prove that $\operatorname{dim}\left(H_{Z}\right)=0$ for a general $H \in \mathcal{H}(-Z)$. Since $W \varsubsetneqq Z_{1}$ and $h^{1}\left(\mathbb{P}^{m}, \mathcal{I}_{Z_{1}}(d)\right)=0$, we have $\mathcal{H}(-W) \neq \emptyset$. Since $W_{\text {red }}=Z_{\text {red }}$ and $Z \subset W$, to prove parts (ii) and (iii) of the lemma it is sufficient to prove $\operatorname{dim}\left(\left(H_{W}\right)_{c}\right)=0$ for a general $H_{W} \in \mathcal{H}(-W)$, where $W$ is as above and $\left(H_{W}\right)_{c}$ is as in Notation 2 Assume that this is not true; therefore

(1) either the contact locus $\left(H_{W}\right)_{c}$ contains a positive-dimensional component $J_{i}$ containing some of the $P_{i}$ 's, for $1 \leq i \leq t-2$,

(2) or the contact locus $\left(H_{W}\right)_{c}$ contains a positive-dimensional irreducible component $T$ containing $Q$.

Set $Z_{3}:=\bigcup_{i=1}^{t-3} 2 P_{i}$ and $Z^{\prime}:=3 O \cup Z_{3}$.

(a) Here we assume the existence of a positive-dimensional component $J_{i} \subset$ $\left(H_{W}\right)_{c}$ containing one of the $P_{i}$ 's, say for example $J_{t-2} \ni P_{t-2}$. Thus a general element of $\left|\mathcal{I}_{W}(d)\right|$ is singular along a positive-dimensional irreducible algebraic set containing $P_{t-2}$. Let $w: M \rightarrow \mathbb{P}^{m}$ denote the blowing-up of $\mathbb{P}^{m}$ at the points $O, P_{1}, \ldots, P_{t-3}$. Set $E_{0}:=w^{-1}(O)$ and $E_{i}:=w^{-1}\left(P_{i}\right), 1 \leq i \leq t-3$. Let $A$ be the only point of $M$ such that $w(A)=P_{t-2}$. For each integer $y \geq 0$ we have $w_{*}\left(\mathcal{I}_{y A} \otimes w^{*}\left(\mathcal{O}_{\mathbb{P}^{m}}(d)\right)\left(-3 E_{0}-2 E_{1}-\cdots-2 E_{t-3}\right)\right)=\mathcal{I}_{Z^{\prime} \cup y P_{t-2}}(d)$. Applying Lemma 1 to the variety $M$, the line bundle $w^{*}\left(\mathcal{O}_{\mathbb{P} m}(d)\right)\left(-3 E_{0}-2 E_{1}-\cdots-2 E_{t-3}\right)$, the point $A$ and the integer $y=2$, we get a contradiction.

(b) Here we prove the non-existence of a positive-dimensional $T \subset\left(H_{W}\right)_{c}$ containing $O$. Let $w_{1}: M_{1} \rightarrow \mathbb{P}^{m}$ denote the blowing-up of $\mathbb{P}^{m}$ at the points $P_{1}, \ldots, P_{t-2}$. Set $E_{i}:=w_{1}^{-1}\left(P_{i}\right), 1 \leq i \leq t-2$. Let $B \in M_{1}$ be the only point of $M_{1}$ such that $w_{1}(B)=O$. For each integer $y \geq 0$ we have

$$
w_{1 *}\left(\mathcal{I}_{y B} \otimes w_{1}^{*}\left(\mathcal{O}_{\mathbb{P}^{m}}(d)\right)\left(-2 E_{1}-\cdots-2 E_{t-2}\right)\right)=\mathcal{I}_{Z^{\prime} \cup y O}(d) .
$$

Since $h^{1}\left(\mathbb{P}^{m}, \mathcal{I}_{Z_{2}}(d)\right)=0$ and $\left|\mathcal{I}_{Z_{2}}(d)\right| \subset\left|\mathcal{I}_{Z}(d)\right|$, by Lemma 1 (with $y=3$ ) we get a contradiction.

In [3, Lemmas 5 and 6$]$, we proved the following two lemmas:

Lemma 3. Fix integers $m \geq 2$ and $d \geq 5$. If $m \leq 4$, then assume $d \geq 6$. Set $\alpha:=\left\lfloor\left(\begin{array}{c}m+d-1 \\ m\end{array}\right) /(m+1)\right\rfloor$. Let $Z_{i} \subset \mathbb{P}^{m}, i=1,2$, be a general union of $i$ triple points and $\alpha-i$ double points. Then $h^{1}\left(\mathcal{I}_{Z_{i}}(d)\right)=0$.

Lemma 4. Fix integers $m \geq 2$ and $d \geq 6$. If $m \leq 4$, then assume $d \geq 7$. Set $\beta:=\left\lfloor\left(\begin{array}{c}m+d-2 \\ m\end{array}\right) /(m+1)\right\rfloor$. Let $Z \subset \mathbb{P}^{m}$ be a general union of one quadruple point and $\beta-1$ double points. Then $h^{i}\left(\mathcal{I}_{Z}(d)\right)=0$.

We will use the following setup.

Notation 3. Fix any $Q \in \tau(X) \backslash X$. For $d \geq 3$ the point $Q$ uniquely determines a point $B \in X$ and (up to a non-zero scalar) a tangent vector $\nu$ of $X$ with $\nu_{\text {red }}=\{B\}$. We have $Q \in\langle\nu\rangle \backslash\{B\}$ and $T_{Q} \tau(X)$ is tangent to $\tau(X) \backslash X$ exactly along the line $\langle\nu\rangle=\langle\{B, Q\}\rangle$. Let $O \in \mathbb{P}^{m}$ be the only point such that $j_{n, d}(O)=B$. Let $u_{O}: \widetilde{X} \rightarrow \mathbb{P}^{m}$ be the blowing-up of $O$. Let $E:=u_{O}^{-1}(O)$ denote the exceptional divisor. For all integers $x, e$ set $\mathcal{O}_{\tilde{X}}(x, e E):=u^{*}\left(\mathcal{O}_{\mathbb{P}}(x)\right)(e E)$. Let $\mathcal{H}$ denote the linear system $\left|\mathcal{O}_{\tilde{X}}(d,-3 E)\right|$ on $\widetilde{X}$. 
Remark 3 . When $d \geq 4$, the line bundle $\mathcal{O}_{\widetilde{X}}(d,-3 E)$ is very ample, $u_{*}\left(\mathcal{O}_{\widetilde{X}}(d,-3 E)\right)$ $=\mathcal{I}_{3 O}(1), h^{0}\left(\widetilde{X}, \mathcal{O}_{\widetilde{X}}(d,-3 E)\right)=\left(\begin{array}{c}m+d \\ m\end{array}\right)-\left(\begin{array}{c}m+2 \\ m\end{array}\right)$ and $h^{i}\left(\widetilde{X}, \mathcal{O}_{\widetilde{X}}(d,-3 E)\right)=0$ for all $i>0$.

Lemma 5. Fix integers $m \geq 2$ and $d \geq 5$. If $m \leq 4$, then assume $d \geq 6$. Set $\alpha:=\left\lfloor\left(\begin{array}{c}m+d-1 \\ m\end{array}\right) /(m+1)\right\rfloor$. Fix an integer $t$ such that $3 \leq t \leq \alpha$. The linear system $\mathcal{H}$ on $\widetilde{X}$ is not $(t-3)$-weakly defective. For a general $O_{1}, \ldots, O_{t-2} \in \widetilde{X}$ a general $H \in\left|\mathcal{H}\left(-2 O_{1}-\cdots-2 O_{t-2}\right)\right|$ is singular only at the points $O_{1}, \ldots, O_{t-2}$ which are ordinary double points of $H$.

Proof. Fix general $O_{1}, \ldots, O_{t-2} \in \tilde{X}$. Fix $j \in\{1, \ldots, t-2\}$ and set $Z^{\prime}:=3 O_{j} \cup$ $\bigcup_{i \neq j} 2 O_{i}, Z^{\prime \prime}:=\bigcup_{i=1}^{t-2} 2 O_{i}$ and $W:=3 O_{j} \cup \bigcup_{i \neq j} 2 O_{i}$. We have $u_{*}\left(\mathcal{I}_{Z^{\prime}}(d,-3 E)\right) \cong$ $\mathcal{I}_{W \cup 3 O}(1)$. The case $i=2$ of Lemma 3 gives $h^{1}\left(\mathcal{I}_{Z}(d,-3 E)\right)=0$. Lemma 1 applied to a blowing-up of $\mathbb{P}^{m}$ at $\left\{O, O_{1}, \ldots, O_{t-2}\right\} \backslash\left\{O_{j}\right\}$ shows that a general $H \in \mathcal{H}(-Z)$ has an isolated singular point at $O_{j}$. Since this is true for all $j \in\{1, \ldots, t-2\}$, $\mathcal{H}$ is not $(t-3)$-weakly defective (just by the definition of weak defectivity). The second assertion follows from the first one and [6, Theorem 1.4].

Now we can apply Lemmas 2, 3, 4 and 5, and get the following result.

Theorem 5. Fix integers $m \geq 2$ and $d \geq 6$. If $m \leq 4$, then assume $d \geq 7$. Set $\beta:=\left\lfloor\left(\begin{array}{c}m+d-2 \\ m\end{array}\right) /(m+1)\right\rfloor$. Fix an integer $t$ such that $3 \leq t \leq \beta+1$. Then $\tau(X, t)$ is not drip defective.

Proof. Fix general $P_{1}, \ldots, P_{t-2}, O \in \mathbb{P}^{m}$ and a general line $L \subset \mathbb{P}^{m}$ such that $O \in L$. Set $Z:=Z(O, L) \cup \bigcup_{i=1}^{t-2} 2 P_{i}, W:=3 O \cup \bigcup_{i=1}^{t-2} 2 P_{t-2}, W^{\prime}:=3 O \cup$ $3 O_{1} \cup \bigcup_{i=2}^{t-2} 2 P_{t-2}$ and $W^{\prime \prime}:=4 O \cup \bigcup_{i=1}^{t-2} 2 P_{t-2}$. Take $O_{i} \in \widetilde{X}$ such that $u_{O}\left(O_{i}\right)=$ $P_{i}, 1 \leq i \leq t-2$. Since $u_{O_{*}}\left(\mathcal{I}_{2 O_{1} \cup \cdots \cup 2 O_{t-2}}(d,-4 E)\right) \cong \mathcal{I}_{W}(d)$, Lemma 4 gives $h^{1}\left(\mathcal{I}_{2 O_{1} \cup \ldots \cup 2 O_{t-2}}(d,-4 E)\right)=0$. Since $Z(O, L) \subset 3 O$, the case $y=3$ of Lemma 1 applied to the blowing-up of $\mathbb{P}^{m}$ at $O_{1}, \ldots, O_{t-2}$ shows that a general $H \in\left|\mathcal{I}_{W}(d)\right|$ has an isolated singularity at $O$ with multiplicity at most 3 .

Recall that $\operatorname{Sing}(\tau(X))=X$ and that for each $Q \in \tau(X) \backslash X$ there is a unique $O \in X$ and a unique tangent vector $\nu$ to $X$ at $O$ such that $Q \in\langle\nu\rangle$ and that $\langle\nu\rangle \backslash\{O\}$ is the contact locus of the tangent space $T_{Q} \tau(X)$ with $\tau(X) \backslash X$.

Let $P$ be a general point of $\tau(X, t)$; i.e. fix a general $\left(P_{1}, \ldots, P_{t-2}, Q\right) \in X^{t-2} \times$ $\tau(X)$ and a general $P \in\left\langle\left\{P_{1}, \ldots, P_{t-2}, Q\right\}\right\rangle$.

Proof of Theorem 1. Fix a general $P \in \tau(X, t)$, say $P \in\left\langle\left\{P_{1}, \ldots, P_{t-2}, Q\right\}\right\rangle$ with $\left(P_{1}, \ldots, P_{t-2}, Q\right)$ general in $X^{t-2} \times \tau(X)$. Terracini's lemma for joins ([1, Corollary 1.10]) gives $T_{P} \tau(X, t)=\left\langle T_{P_{1}} X \cup \cdots \cup T_{P_{t-2}} X \cup T_{Q} \tau(X)\right\rangle$. Let $O$ be the point of $\mathbb{P}^{m}$ such that $Q \in T_{j_{m, d}(O)} X$. Let $\mathcal{H}^{\prime}$ (resp. $\mathcal{H}^{\prime \prime}$ ) be the set of all hyperplanes $H \subset \mathbb{P}^{N}$ containing $T_{Q} \tau(X)$ (resp. $T_{P} \tau(X, t)$ ). We may see $\mathcal{H}^{\prime}$ and $\mathcal{H}^{\prime \prime}$ as linear systems on the blowing-up $\widetilde{X}$ of $\mathbb{P}^{m}$ at $O$. Take $O_{i} \in \widetilde{X}, 1 \leq i \leq t-2$, such that $P_{i}=u\left(O_{i}\right)$ for all $i$. We have $\mathcal{H}^{\prime \prime}=\mathcal{H}^{\prime}\left(-2 P_{1}-\cdots-2 P_{t-2}\right)$ and $\mathcal{H} \subseteq \mathcal{H}^{\prime}$, where $\mathcal{H}$ is defined in Notation 3. Since $\left(P_{1}, \ldots, P_{t-2}\right)$ is general in $X^{t-2}$ for a fixed $Q$ and $\mathcal{H} \subseteq \mathcal{H}^{\prime}$, Lemma 5 gives that a general $H \in \mathcal{H}^{\prime \prime}$ intersects $X$ in a divisor which, outside $O$, is singular only at $P_{1}, \ldots, P_{t-2}$ and with an ordinary node at each $P_{i}$. Now assume $P \in\left\langle\left\{P_{1}^{\prime}, \ldots P_{t-2}^{\prime}, Q^{\prime}\right\}\right\rangle$ for some other $\left(P_{1}^{\prime}, \ldots, P_{t-2}^{\prime}, Q^{\prime}\right) \in X^{t-2} \times \tau(X)$. Since $P$ is general in $\tau(X, t)$ and $\tau(X, t)$ has the expected dimension, the $(t-1)$ uple $\left(P_{1}^{\prime}, \ldots, P_{t-2}^{\prime}, Q^{\prime}\right)$ is general in $X^{t-2} \times \tau(X)$. Hence $H \cap X$ is singular at 
each $P_{i}^{\prime}, 1 \leq i \leq t-2$, and with an ordinary node at each $P_{i}^{\prime}$. Since $O$ is not an ordinary node of $H \cap X$, we get $\left\{P_{1}, \ldots, P_{t-2}\right\}=\left\{P_{1}^{\prime}, \ldots, P_{t-2}^{\prime}\right\}$. Thus $O=O^{\prime}$. Hence $H$ is tangent to $\tau(X)_{\text {reg }}$ exactly along the line $\langle\{Q, O\}\rangle \backslash\{O\}$. Hence $Q^{\prime} \in\langle\{Q, O\}\rangle$. Assume $Q \neq Q^{\prime}$. Since $P$ is general in $\tau(X, t)$, then $P \notin \tau(X, t-1)$. Hence $Q^{\prime} \notin\left\langle\left\{P_{1}, \ldots, P_{t-2}\right\}\right\rangle$ and $Q \notin\left\langle\left\{P_{1}, \ldots, P_{t-2}\right\}\right\rangle$. Thus $\left\langle\left\{P_{1}, \ldots, P_{t-2}, Q\right\}\right\rangle \cap\left\langle\left\{P_{1}, \ldots, P_{t-2}, Q^{\prime}\right\}\right\rangle=\left\langle\left\{P_{1}, \ldots, P_{t-2}\right\}\right\rangle$ if $Q \neq Q^{\prime}$. Since $P \in\left\langle\left\{P_{1}, \ldots, P_{t-2}, Q\right\}\right\rangle \cap\left\langle\left\{P_{1}, \ldots, P_{t-2}, Q^{\prime}\right\}\right\rangle$, we get a contradiction.

Proof of Theorem 4. The case $t=2$ is well known and follows from the facts that for any $O \in X$ and any $Q \in T_{O} X \backslash\{O\}$ the group $G_{O}:=\left\{g \in \operatorname{Aut}\left(\mathbb{P}^{n}\right): g(O)=O\right\}$ acts on $T_{O} X$ and the stabilizer $G_{O, Q}$ of $Q$ for this action is the line $\langle\{O, Q\}\rangle$, while $T_{O} X \backslash\langle\{O, Q\}\rangle$ is another orbit for $G_{O, Q}$. Thus we may assume $t \geq 3$. Fix a general $P \in \tau(X, t)$ and a general hyperplane $H \supset T_{P} \tau(X, t)$. If $H$ is tangent to $\tau(X)$ at a point $Q^{\prime} \in \tau(X) \backslash X$, then it is tangent along a line containing $Q^{\prime}$. Let $E \in X$ be the only point such that $Q^{\prime} \in T_{E} X$. We get $T_{E} X \subset \tau(X, t)$ and that $H \cap T_{E} X$ is larger than the double point $2 E \subset X$. Theorem 1 gives that $Q, Q^{\prime}$ and $E$ are collinear; i.e. $H$ is tangent only along the line $\nu$.

\section{REFERENCES}

[1] B. Ådlandsvik, Joins and higher secant varieties. Math. Scand. 61 (1987), no. 2, 213-222. MR947474 (89j:14030)

[2] E. Ballico, On the weak non-defectivity of Veronese embeddings of projective spaces. Cent. Eur. J. Math. 3 (2005), no. 2, 183-187. MR2129920 (2005m:14097)

[3] E. Ballico, A. Bernardi, Partial stratification of secant varieties of Veronese varieties via curvilinear subschemes, arXiv:1010.3546v1 [math.AG].

[4] A. Bernardi, A. Gimigliano, M. Idà. Computing symmetric rank for symmetric tensors. J. Symb. Comput. 46 (2011) 34-53. MR2736357

[5] M. V. Catalisano, A. V. Geramita, A. Gimigliano, On the secant varieties to the tangential varieties of a Veronesean. Proc. Amer. Math. Soc. 130 (2002), no. 4, 975-985. MR 1873770 (2002m:14042)

[6] L. Chiantini, C. Ciliberto, Weakly defective varieties. Trans. Amer. Math. Soc. 454 (2002), no. 1, 151-178. MR.1859030 (2003b:14063)

[7] L. Chiantini, C. Ciliberto, On the dimension of secant varieties. J. Eur. Math. Soc. 12 (2010), no. 5, 1267-1291. MR2677616

[8] C. Ciliberto, F. Russo, Varieties with minimal secant degree and linear systems of maximal dimension on surfaces. Adv. Math. 206 (2006), no. 1, 1-50. MR2199628 (2007d:14097)

[9] D. Eisenbud, Commutative algebra. With a view toward algebraic geometry. Graduate Texts in Mathematics, 150. Springer-Verlag, New York, 1995. MR1322960(97a:13001)

[10] M. Mella, Singularities of linear systems and the Waring problem. Trans. Amer. Math. Soc. 358 (2006), no. 12, 5523-5538. MR2238925 (2007h:14059)

[11] M. Mella, Base loci of linear systems and the Waring problem. Proc. Amer. Math. Soc. 137 (2009), no. 1, 91-98. MR2439429 (2009g:14072)

Department of Mathematics, University of Trento, 38123 Povo (TN), Italy

E-mail address: ballico@science.unitn.it

GALAAD, INRIA Méditerranée, BP 93, 06902 Sophia Antipolis, France

E-mail address: alessandra.bernardi@inria.fr 